Cite this: Phys. Chem. Chem. Phys., 2013 15, 14660

Received 14th May 2013,

Accepted 24th June 2013

DOI: $10.1039 /$ c3cp52026a

www.rsc.org/pccp

\section{Exploiting level anti-crossings for efficient and selective transfer of hyperpolarization in coupled nuclear spin systems $\dagger$}

\author{
Andrey N. Pravdivtsev, ${ }^{\text {ab }}$ Alexandra V. Yurkovskaya, ${ }^{\text {ab }}$ Robert Kaptein, ${ }^{\text {bc }}$ \\ Karsten Miesel, ${ }^{d}$ Hans-Martin Vieth ${ }^{d}$ and Konstantin L. Ivanov ${ }^{* a b}$
}

\begin{abstract}
Spin hyperpolarization can be coherently transferred to other nuclei in field-cycling NMR experiments. At low magnetic fields spin polarization is redistributed in a strongly coupled network of spins. Polarization transfer is most efficient at fields where level anti-crossings (LACs) occur for the nuclear spin-states. A further condition is that field switching to the LAC positions is non-adiabatic in order to convert the starting population differences into spin coherences that cause time-dependent mixing of states. The power of this method has been demonstrated by studying transfer of photo-Chemically Induced Dynamic Nuclear Polarization (photo-CIDNP) in N-acetyl-tryptophan. We have investigated the magnetic field dependence and time dependence of coherent CIDNP transfer and directly assessed nuclear spin LACs by studying polarization transfer at specific field positions. The proposed approach based on LACS is not limited to CIDNP but is advantageous for enhancing NMR signals by spin order transfer from any type of hyper-polarized nuclei.
\end{abstract}

\section{Introduction}

NMR is a powerful and versatile spectroscopic technique with an abundance of applications in physics, chemistry, biology and medicine. However, the technique suffers from a low inherent sensitivity, which is a limiting factor in many applications. The sensitivity problem comes from the poor Boltzmann polarization of nuclear spins at thermal equilibrium. A way to overcome this problem is using non-thermal spin polarization, also called hyperpolarization. Among the techniques based on this approach are Dynamic Nuclear Polarization (DNP), ${ }^{1-4}$ Chemically Induced Dynamic Nuclear Polarization $(\mathrm{CIDNP})^{5}$ and Para-Hydrogen Induced Polarization (PHIP). ${ }^{6-8}$ All these methods employ strongly non-thermally polarized spins, thus allowing one to enhance the NMR signal intensity by several orders of magnitude.

Although hyperpolarization techniques made numerous new NMR applications possible, ${ }^{6,9-17}$ there are still many

\footnotetext{
${ }^{a}$ International Tomography Center SB RAS, Novosibirsk, 630090, Russia. E-mail: ivanov@tomo.nsc.ru; Fax: +7-383-333-1399; Tel: +7-383-333-3861

${ }^{b}$ Novosibirsk State University, Novosibirsk, 630090, Russia

${ }^{c}$ Utrecht University, NL-3584 CH, Utrecht, The Netherlands

${ }^{d}$ Freie Universität Berlin, Berlin, 14195, Germany

† Electronic supplementary information (ESI) available: Experimental data for low-field CIDNP transfer and $T_{1}$-relaxation, Fig. S1-S3 and Table S1. See DOI: 10.1039/c3cp52026a
}

problems to be solved in this field. In particular, it is of importance to further develop strategies for optimizing the hyperpolarization process and the transfer of polarization from the primarily polarized spins to the target nuclei of choice. This step is required, for instance, in PHIP experiments to hyperpolarize other nuclei in the molecule by spin order transfer from the two protons prepared in their singlet state.

In the present contribution we will address the problem of polarization transfer within scalar coupled homonuclear systems in field-cycling NMR experiments. We propose that highly efficient and selective hyperpolarization transfer based on coherent spin mixing can be achieved by exploiting spin coherences formed in the vicinity of nuclear spin Level Anti-Crossing (LAC) points. LAC is a well-known concept in physics; ${ }^{18}$ previously LACs have been used in optical nuclear polarization $^{19-21}$ and in solid-state DNP. ${ }^{22,23}$ Here we will deal with liquid samples where the systematic consideration of LACs provides a powerful resource for manipulating the transfer of spin hyperpolarization in PHIP and in CIDNP. The advantage of LACs in polarization transfer is two-fold: (i) they provide a high polarization transfer efficiency (much higher than, for instance, incoherent processes such as cross-relaxation) and (ii) they enable highly selective transfer, which proceeds only between certain types of spin order. Previously, pronounced features in the CIDNP field dependence due to LACs were reported ${ }^{24}$ and 
they have also been considered in PHIP. ${ }^{25-27}$ However, polarization transfer kinetics was never studied in detail and nor was the coherent nature of the transfer efficiently exploited.

To form coherences between crossing nuclear spin levels we will use NMR with fast field-cycling. One way ${ }^{27,28}$ to form spin coherences in field-cycling NMR experiments is by polarizing a spin system at high field and subsequently transferring it to the LAC field. When the field switching is non-adiabatic the initial population differences are transferred into spin coherences. Their evolution results in a polarization redistribution, which is particularly fast and efficient. More specifically, we will show that using LACs it is possible to exactly exchange the populations of the crossing levels thereby reaching a transfer of $100 \%$ efficiency for a particular spin order as long as relaxation effects are negligible; in the presence of relaxation the fraction of polarization transfer still remains high. After the evolution of spin coherences has taken place the system is brought to the high NMR detection field where the polarized signals are measured. Our previous studies have shown that this method of creating spin coherences without direct NMR excitation is feasible; moreover, by setting the field to specific LAC positions and controlling the speed of field variation one can manipulate the transfer process. To form hyperpolarization a useful method is photo-CIDNP in reversible photoreactions. An advantage of photo-CIDNP is that timing of the chemical reactions can be precisely controlled; in addition, the method gives NMR enhancement of the order of 100-200 (ref. 29). Thus, initially some of the NMR lines are strongly enhanced, while the others are enhanced only weakly. This is in contrast to, for instance, the solidstate DNP case where all NMR lines of the sample exhibit virtually the same enhancement. We will show that LACs enable transferring polarization between strongly and weakly polarized spins to enhance particular lines in the NMR spectrum.

Experimental results will be reported for the amino acid $\mathrm{N}$-acetyl-tryptophan ( $\mathrm{N}$-Ac-Trp), which has two different networks of four scalar coupled protons each. In the $N$-Ac-Trp molecule the starting polarizations ${ }^{30}$ of the individual protons are different due to differences in the hyperfine interactions in the $N$-Ac-Trp cation radical. Low-field experiments make it possible to redistribute the initial polarizations by a coherent transfer process. The experimental results will be supported by accurate theoretical calculations, which are a prerequisite for a quantitative understanding of the experimental findings and for the design of new experiments.

\section{Methods}

\section{A. Materials}

$N$-Ac-Trp was used as received from Bachem. The dye 4-carboxybenzophenone (4-CBP), the solvent $\mathrm{D}_{2} \mathrm{O}, \mathrm{DCl}$ and $\mathrm{NaOD}$ were purchased from Sigma-Aldrich. DCl and NaOD were used to adjust the $\mathrm{pH}$. In the experiments the following aqueous solution was used: $2 \mathrm{mM} \mathrm{N-Ac-Trp}$ with $1 \mathrm{mM} 4$-CBP at pH 11.0. NMR parameters (chemical shifts, scalar spin-spin couplings and high-field $T_{1}$-relaxation times) that are needed for modeling the polarization transfer effects are given in Table S1 (see ESI $\dagger$ ). The chemical structure of $N$-Ac-Trp is given

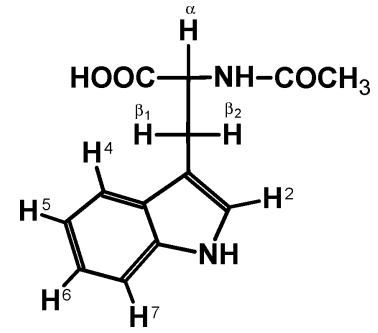

Chart 1 Structure of $N$-acetyl-tryptophan ( $N$-Ac-Trp) with numbering and nomenclature of the protons.

in Chart 1. CIDNP was formed by means of photo-induced electron transfer from $N$-Ac-Trp to 4 -CBP. ${ }^{30}$

\section{B. Field-cycling NMR}

The field-cycling setup is based on mechanical shuttling of the NMR probe-head between positions of different field strength as described in detail in an earlier publication. ${ }^{31}$ This setup allows for the combination of fast field-cycling (less than $0.3 \mathrm{~s}$ for shuttling the probe-head between the highest and the lowest magnetic field) with high-resolution NMR detection (NMR linewidth is about $0.3 \mathrm{~Hz}$ ). NMR detection is done at a field $B_{0}=7$ Tesla under permanent sample rotation $(20 \mathrm{~Hz})$. The evolution of the polarized spins can be studied at a variable field $B_{\text {int }}$ ( $c f$. Fig. 1) in the range between $50 \mu \mathrm{T}$ and $7 \mathrm{~T}$. In the range of 0.1 to $7 \mathrm{~T}$ the field is set by mechanical positioning of the NMR probe with the sample in the fringe field of the spectrometer cryo-magnet, while below $0.1 \mathrm{~T}$ the field is set by controlling the current of an auxiliary magnetic system placed under the bore of the NMR magnet at a position where the fringe field is about $50 \mathrm{mT}$. The auxiliary magnet provides a magnetic field ranging from $-50 \mathrm{mT}$ to $50 \mathrm{mT}$, which is superimposed on the NMR fringe field at the lowest sample position. The current in the auxiliary magnet is pre-set for each field-cycling experiment and is changed only between subsequent experiments in order to vary the $B_{\text {int }}$ field. Thus, the

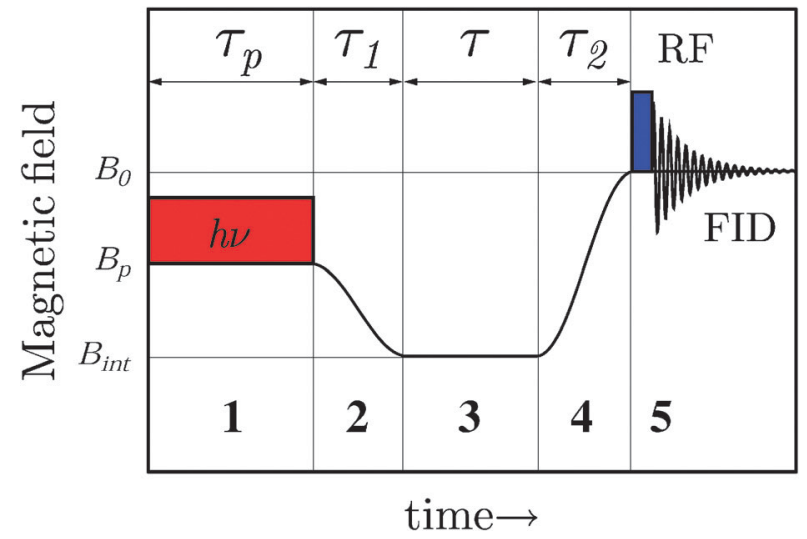

Fig. 1 Protocol of the polarization transfer experiment with 5 stages: CIDNP preparation by light (stage 1 , duration $\tau_{\mathrm{p}}$ ) at $B=B_{\mathrm{p}}$; field variation to $B=B_{\text {int }}$ (stage 2, duration $\tau_{1}$ ); polarization transfer at $B_{\text {int }}$ (stage 3 , duration $\tau$ ); field variation to the detection field $B=B_{0}$ (stage 4, duration $\tau_{2}$ ); Fourier-transform NMR detection (stage 5). 
system allows setting the field in the range from $0.05 \mathrm{mT}$ to $0.1 \mathrm{~T}$. Additional coils also minimize the gradient of the $B_{\text {int }}$ field resulting in a field variation along the sample of less than $0.1 \mathrm{mT}$ for the low-field part. Since the field switching is digitally controlled its time profile, $B(t)$, is known precisely. The setup allows generating CIDNP at any magnetic field by light irradiation via a liquid light-guide. ${ }^{31}$

\section{Experimental protocol}

The experimental protocol depicted in Fig. 1 consists of 5 consecutive stages. During stage 1 (preparation) CIDNP is generated at $B=B_{\mathrm{p}}$ by using light irradiation. In our experiments we always set $B_{\mathrm{p}}$ equal to the NMR detection field $B_{0}$ with the advantage that the starting polarization of the spins at the end of stage 1 is exactly known from the high-field CIDNP spectrum. Immediately after CIDNP formation the probe is rapidly moved to the intermediate field value $B_{\text {int }}$ (stage 2) during the time period $\tau_{1}$. Then after a period of free evolution of variable duration $\tau$ (stage 3 ) the probe is rapidly moved back (stage 4) to $B_{0}$ during the time period $\tau_{2}$. Finally, at $B=B_{0}$ the Fourier-transform NMR spectrum is obtained (stage 5 ). The unique combination of fast field-cycling with high resolution NMR $(\approx 0.3 \mathrm{~Hz})$ enables measuring the NMR line intensities of all individual protons in $N$-Ac-Trp. Thus, it is possible to study the CIDNP transfer phenomena and measure the CIDNP time evolution for all coupled protons.

We performed a detailed study of CIDNP transfer by varying $B_{\text {int }}$ and the experimental timing over a wide range. This allowed us to identify the role of LACs and to optimize the transfer efficiency. The theory of low-field polarization transfer is outlined in the following section.

\section{Theory}

Coherent polarization transfer effects are described for the conditions: (i) strong coupling of spins at low magnetic fields and (ii) non-adiabatic field switching. Strong coupling results in simultaneous polarization of several spins, while non-adiabatic changes of the Hamiltonian allow for transferring state population differences into coherences and vice versa.

The condition of strong (or weak) coupling is determined by the relation between the difference, $\delta \nu$, in Zeeman interaction of the spins and their spin-spin interaction, $J$. Spins are considered strongly coupled when $\delta \nu$ is smaller than or comparable to $J$; the opposite situation $\delta \nu \gg J$ corresponds to weak coupling. As $\delta \nu$ is directly proportional to the external magnetic field the situation of strongly coupled spins corresponds to the low-field case, while at sufficiently high field the spins are weakly coupled.

The concept of strong coupling is important for our work because strongly coupled spins always have collective (mixed) eigen-states. As a consequence, polarization transfer ${ }^{32,33}$ takes place: by polarizing one spin one also polarizes the other. Let us consider a two-spin system as an example. In a two-spin system polarization transfer occurs via coherent mixing of eigen-states characterized by zero $z$-projection of the total spin, $I_{z}$. At high field these eigen-states are, obviously, $\alpha \beta$ and $\beta \alpha$, i.e., products of single-spin Zeeman states $\alpha$ and $\beta$. However, at $B=0$ the eigen-states are the mixed singlet (S) and triplet $\left(\mathrm{T}_{0}\right)$ states. The splitting between the levels, $\delta E$, is equal to $\delta E=\sqrt{J^{2}+(\delta \nu)^{2}}$. At $\delta \nu=0$ (i.e., zero magnetic field) it is minimal and equal to $|J|$. This situation can be considered a LAC, also called avoided level crossing: at $B=0$ the two levels tend to cross but the coupling matrix element splits them by $|J|$ (Fig. 2a). In the vicinity of the LAC the spins are most strongly coupled; in addition, coefficients which describe the mixing of $\alpha \beta$ and $\beta \alpha$ in the eigen-states experience abrupt changes upon going to the crossing point. The notions of LAC and 'strong coupling' are interrelated: in fact, for the simplest two-spin system the LAC and the strongest coupling are both occurring at zero field. For two spins there is only one LAC, which occurs for the states with $I_{z}=0$; it is always located at zero field. For larger spin systems there are additional LACs at $B=B_{\text {lac }} \neq 0$. For instance, in a three-spin system there is one such LAC (see Fig. 2b). In general, when one passes through a LAC the mixing of spin states changes significantly also resulting in abrupt changes in the polarization transfer efficiency. ${ }^{33}$ For this reason the notion of LAC takes a central position in our work.

Strong coupling of spins is necessary but not sufficient for CIDNP transfer in our experiments. This is because initially the spin system is hyperpolarized at high field where the spins keep their individual spin order. For polarization transfer it is crucial how fast the magnetic field is switched; more specifically, the field variation has to be non-adiabatic. In general, for two eigen-states, $|i\rangle$ and $|j\rangle$, of a time-dependent Hamiltonian the adiabaticity condition is described ${ }^{34}$ by the parameter $\xi_{\mathrm{ij}}(t)=$ $\left|\alpha_{\mathrm{ij}}(t) / 2 \pi \delta E_{\mathrm{ij}}(t)\right|$ where $\alpha_{\mathrm{ij}}(t)=\langle\mathrm{i}|(\mathrm{d}|\mathrm{j}\rangle / \mathrm{d} t)$ describes how the

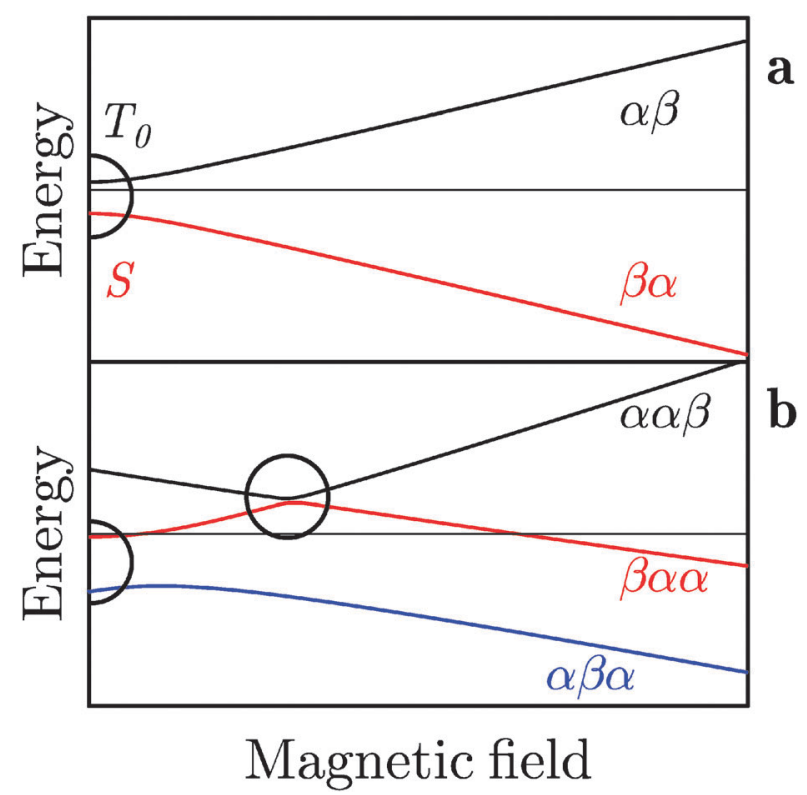

Fig. 2 Schematic description of LACs for a two-spin system (a) and a three-spin system (b). LACs are indicated by circles, spin levels are assigned at high fields and in the case of (a) also at zero field. In the case of (b) to clearly visualize the LACs we subtracted the large Zeeman energy $\hbar \gamma \beta I_{z}$ from the total energy. 
eigen-states of the system change with time and $\delta E_{\mathrm{ij}}(t)$ is the time-dependent splitting between the levels given in $\mathrm{Hz}$. When $\alpha_{\mathrm{ij}}(t) \ll 2 \pi \delta E_{\mathrm{ij}}(t)$ also $\xi_{\mathrm{ij}}(t) \ll 1$ and the eigen-states change slowly, such that the populations follow the adiabatic states of the Hamiltonian. In the opposite case the Hamiltonian changes abruptly with the result that the density matrix of the spin system has to be projected on the new eigen-basis; consequently, the state populations are mixed and, additionally, coherences between them can be formed. In the case of two spins $\frac{1}{2}$ the eigen-states of the system with $I_{z}=0$ at arbitrary field (adiabatic states) are

$$
\begin{gathered}
|1\rangle=\cos \theta|\alpha \beta\rangle+\sin \theta|\beta \alpha\rangle \\
|2\rangle=-\sin \theta|\alpha \beta\rangle+\cos \theta|\beta \alpha\rangle
\end{gathered}
$$

where $\tan 2 \theta=J / \delta \nu$; these states change from $\alpha \beta$ and $\beta \alpha$ at high field to $\mathrm{S}$ and $\mathrm{T}_{0}$ at low field. Consequently, the adiabaticity parameter is

$$
\xi_{12}(t)=\frac{1}{2 \pi \delta E(t)} \cdot \frac{\mathrm{d} \theta(t)}{\mathrm{d} t}
$$

Thus, the adiabaticity condition is determined by how fast the mixing angle, $\theta(t)$, changes upon field variation as compared to $\delta E(t)=\sqrt{J^{2}+(\delta \nu(t))^{2}}$. To estimate the efficiency of mixing after passing through an isolated LAC at a constant speed one can use the Landau-Zener theory, which gives the following transition probability ${ }^{35}$ for the populations of the two coupled states

$$
P_{12}=\exp \left(-\frac{(2 \pi J)^{2}}{\mathrm{~d}(\delta \nu) / \mathrm{d} t}\right)
$$

Here $\mathrm{d}(\delta \nu) / \mathrm{d} t$ gives the rate at which $\delta \nu$ changes during the passage. This expression gives a reasonable estimate for the degree of non-adiabaticity; however, in our case it can hardly be used in practice. This is because we are interested not only in the transition probability but also in the formation of coherences upon fast changes of the Hamiltonian. Besides this, in our experiments we never had a linear variation of $\delta \nu$ upon the field variation but a more complex $B(t)$ time profile. ${ }^{36}$ For this reason, to model experiments on the quantitative level it became necessary to solve numerically the Liouville-von Neumann equation for the density matrix (see below). It is also important to emphasize that although exciting spin coherences by field variation is a rather demanding task our experimental setup makes it feasible, particularly when the spin system has LACs.

To explain the transfer mechanism let us take the example of a hyperpolarized two-spin system, shown in Fig. 3. Let us assume that at high field one of the spins has a positive net CIDNP equal to $M$, while the other spin is not polarized. Such a spin order results in different population of the eigen-states with $I_{z}=0$, which are the $\alpha \beta$ - and $\beta \alpha$-states. When the magnetic field is instantaneously reduced to $B_{\text {int }}=0$ the difference in polarizations is converted into the coherence, $\rho_{\mathrm{ST}_{0}}$, between the

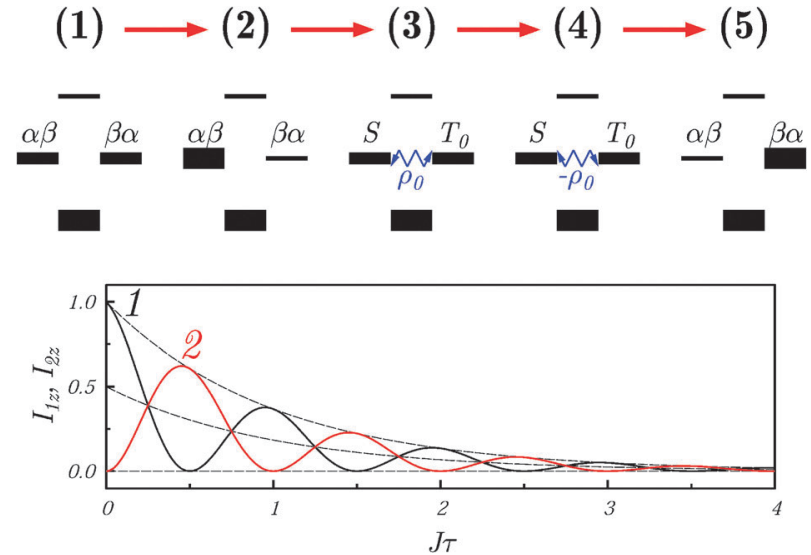

Fig. 3 Scheme of CIDNP transfer in a field-cycling NMR experiment. Top: representation of the CIDNP formation and evolution. Bottom: net polarizations of spins 1 and 2 as oscillatory functions of time interval $\tau$ at zero field; decay of polarization and coherence is taken into account. In the scheme the following state population patterns (PP) (top) are shown. First, spins have the same z-magnetizations (PP 1); after the CIDNP formation net polarizations of spins are $M_{1}=M_{1} M_{2}=0$ (PP 2). Fast jump to low field converts the population difference between the $\alpha \beta$ - and $\beta \alpha$-states into ZQC, $\rho_{\mathrm{ST}_{0}}=\rho_{0}$, between the lowfield $S$ - and $T_{0}$-states (PP 3). Subsequent evolution during the time $\tau=1 / 2 \mathrm{~J}$ results in sign inversion of the ZQC (PP 4); finally, after the second switching to high field spin magnetizations are inverted (PP 5): $M_{1}=0, M_{2}=M$. Magnetizations as a function of $J \tau$ show oscillations, which are damped due to relaxation.

new eigen-states (singlet-triplet states), the Zero-Quantum Coherence (ZQC). At the same time the populations of the $\mathrm{S}$ and $\mathrm{T}_{0}$ states become equal in contrast to those of the $\alpha \beta$ - and $\beta \alpha$-states. Thus, after sudden field switching the initial population difference is completely converted into spin coherence in the new eigen-basis. This coherence immediately starts oscillating with frequency $J$. When after a period of free evolution, $\tau$, the field is switched back to $B=B_{0}$ the ZQC is converted back into the difference in magnetization of the two spins. When the delay is appropriately set $(J \cdot \tau=1 / 2)$ the coherence has the opposite phase and, consequently, the spins exactly exchange their polarization. In this way CIDNP can be transferred between coupled spins in fast field-cycling experiments. The resulting magnetizations of spins oscillate as function of time $\tau$ (Fig. 3). In practice, because of relaxation, the amplitude of the oscillations decays exponentially with time while the level of polarization decreases in parallel.

The spin dynamics can also be described in the framework of the product operator formalism. ${ }^{37}$ Prior to the first field variation the spin system has a spin order given by the operator $\left(\hat{I}_{1 z}-\hat{I}_{2 z}\right)$, which does not change with time at high field as it commutes with the high-field Hamiltonian. After the field switch, however, it is converted to the ZQC between the S and $\mathrm{T}_{0}$ states and is no longer conserved: $J$-coupling mixes it with another spin order, namely, with $i\left(2 \hat{I}_{1 y} \hat{I}_{2 x}-2 \hat{I}_{1 x} \hat{I}_{2 y}\right)$ because $\left[\hat{I}_{1 z}-\hat{I}_{2 z}, J\left(\hat{\boldsymbol{I}}_{1} \cdot \hat{\boldsymbol{I}}_{2}\right)\right]=\mathrm{i} J\left(2 \hat{I}_{1 y} \hat{I}_{2 x}-2 \hat{I}_{1 x} \hat{I}_{2 \mathrm{y}}\right)$. As a consequence, the ZQC evolves as follows: $\mathrm{ZQC}(t)=\left(\hat{I}_{1 z}-\hat{I}_{2 z}\right) \cos 2 \pi J \tau+\left(2 \hat{I}_{1 y} \hat{I}_{2 x}-\right.$ $\left.2 \hat{I}_{1 x} \hat{I}_{2 y}\right) \sin 2 \pi J \tau$. The second field variation makes $\left(\hat{I}_{1 z}-\hat{I}_{2 z}\right) \cos 2$ $\pi J \tau$ a time-independent operator again; the corresponding spin order is then measured by NMR. Thus, the difference in 
magnetizations oscillates as a function of $\tau$; the oscillation frequency is equal to $J$.

As has been pointed out, an important issue is the rate of field variation: for a slow (adiabatic) field switch the spin energy levels keep their initial populations and no coherences occur in the system. Consequently, the state populations after two field switches are exactly the same as before the switching (assuming that relaxation effects are negligible). In contrast, non-adiabatic (sudden) field variation enables the excitation of spin coherences resulting in exchange of population between the chosen pair of levels. Excitation of a specific coherence (for instance, by stepping through a particular $\mathrm{LAC}^{25-28}$ ) also allows for the selective transfer between particular spin orders. As will be shown below, this methodology is applicable for more than two coupled spins although the physics becomes more complex. For instance, for more than two coupled energy levels (which is the case for more than two spins) one can excite two or more coherences from the initial population differences. However, when one steps through an isolated LAC often only a single coherence is excited with high efficiency. In this case the situation is physically similar to the population-coherence exchange in a two-level system.

For simulating the experimental results we used essentially the same method as before. ${ }^{27,28}$ We solved the Liouville-von Neumann equation for the spin density matrix, $\hat{\rho}$, during stages 2, 3 and 4 . The Hamiltonian, $\hat{H}$, of the spin system describes a system of $K$ scalar coupled spin $1 / 2$ nuclei (protons in our case) and includes the Zeeman interaction of spins with the external field and scalar spin-spin coupling. The Hamiltonian is timedependent during stages 2 and 4 because the magnetic field varies with time, but is constant during stage 3 . The Zeeman interaction of the $i$-th spin is conditioned by its chemical shift, $\delta_{i}$; the coupling constants $J_{\mathrm{ij}}$ are specified for all pairs of interacting spins. All the parameters $\delta_{i}$ and $J_{\mathrm{ij}}$ were determined from simulation of the high-resolution NMR spectra taken at high field, $B_{0}=7$ Tesla (see Table S1, ESI $\dagger$ ).

To describe damping of the oscillations in the time dependence of the polarization transfer and the overall decay of hyperpolarization in the system we also considered spin relaxation. For the sake of simplicity, this was done only for stage 3 because the field variation times, $\tau_{1}$ and $\tau_{2}$, were considerably shorter than the typical relaxation times in the system. The time between the field switches, $\tau$, was varied over a wide range; hence, taking relaxation into account was necessary for the period of free evolution at the intermediate field. Spin relaxation was described by the Redfield theory with the full relaxation super-operator $\hat{\hat{R}}$. The relaxation of spins was treated according to the model of fluctuating local fields. This simple model requires as input parameters only the high-field $T_{1^{-}}$and $T_{2}$-relaxation times of the individual spins; we also assumed $T_{1 i}=T_{2 i}$. The relaxation times $T_{1 i}$ were determined at $B=B_{0}$ by the standard inversion-recovery experiment. Although the model chosen cannot take into account more specific effects (such as long-lived states ${ }^{38,39}$ resulting from dipolar relaxation of spins) it gives a realistic description of the decay of spin order (population differences and coherences) in the whole range of magnetic fields.
Finally, the initial condition for the density matrix after the preparation (at $t=\tau_{\mathrm{p}}$ ) is characterized by a set of net polarizations, $M_{i}$, of the individual spins. For the sake of simplicity we neglect other spin orders, for instance, multiplet polarization of the type $\hat{I}_{i z} \hat{I}_{i z}$, a situation that is realistic for high-field CIDNP experiments. Since we are not dealing with multiple-spin orders, we will hereafter use the simpler term 'polarization' instead of the more rigorous terms 'spin order' or 'spin alignment'. Indeed, in our situation it is possible to characterize the system by net magnetizations of spins measured at high field only, which will be referred to as net 'polarization'. Likewise, the term 'polarization transfer' describes the transfer of net magnetization between the coupled spins. At low field differences in net polarization $\left(M_{i}-M_{j}\right)$ are converted into the corresponding spin coherences, which mediate polarization transfer according to the mechanism described above. Values for $M_{i}$ were determined from the CIDNP spectrum measured at $B_{0}=7$ Tesla.

\section{Results and discussion}

The N-Ac-Trp molecule represents a particularly interesting case because there are two subsystems of four coupled protons with different coupling networks, while spin-spin interactions between the protons of the different groups are negligible. The first network (hereafter called Subsystem 1) consists of the $\alpha-\mathrm{CH}$ proton, the $\beta-\mathrm{CH}_{2}$ protons and the aromatic proton in the $\mathrm{H} 2$ position. The second network (Subsystem 2) is formed by the four protons of the six-membered ring of $N$-Ac-Trp.

In Subsystem 1 at low field $B_{\text {int }} \lesssim 100 \mathrm{mT}$ all four spins become strongly coupled; as the field is above $100 \mathrm{mT}$, the three-spin system composed of the $\alpha$-CH proton and the $\beta-\mathrm{CH}_{2}$ protons becomes only weakly coupled to the $\mathrm{H} 2$ proton. In Subsystem 2 each of the four protons has considerable couplings to its nearest neighbors, while all other couplings in this system are small. It is of interest to check whether in Subsystem 2 polarization is transferred only among the neighboring spins or also among remote spins. The latter case is possible because at low fields each pair of neighbors is strongly coupled. As a consequence, the whole system is coupled strongly, including even spins having no coupling to each other. From our previous theoretical studies ${ }^{33}$ we anticipate that under such conditions polarization can be transferred even among spins having no direct couplings.

The CIDNP spectra of each of the two subsystems contains protons with different values $M_{i}$ of polarization, which is a necessary prerequisite for creating ZQCs by fast field variation. At the end of the preparation period, the two $\beta-\mathrm{CH}_{2}$ protons have negative polarization, the $\mathrm{H} 2, \mathrm{H} 4, \mathrm{H} 6$ protons have strong positive polarization, whereas CIDNP of the other protons is relatively small (see Table S1, ESI†).

Let us first consider CIDNP transfer in the four-spin system composed of the $\alpha-\mathrm{CH}, \beta-\mathrm{CH}_{2}$ and $\mathrm{H} 2$ protons. At low fields the entire system is strongly coupled; thus, CIDNP transfer occurs not only among the $\alpha-\mathrm{CH}$ and $\beta-\mathrm{CH}_{2}$ protons but involves the $\mathrm{H} 2$ proton as well. At $B_{\text {int }}=0$ we have found selective coherent 
CIDNP transfer between the $\beta 1-\mathrm{CH}_{2}$ proton and the $\mathrm{H} 2$ proton (see Fig. S1, ESI $\dagger$ ). The origin of the selectivity is described in detail in ESI. $\dagger$ It is, however, more illustrative to consider CIDNP transfer caused by an isolated LAC. To this end we studied polarization transfer at higher fields where pronounced LAC effects can be observed.

As the field increases, the coupling of the $\mathrm{H} 2$ proton to the other three protons becomes weak. Thus, with regard to polarization transfer the subsystem changes to a strongly-coupled three-spin system. The field dependence of CIDNP of the $\alpha-\mathrm{CH}$ proton after two field jumps is shown in Fig. 4a together with the theoretical simulation. In this dependence there is a sharp dip reflecting transfer of negative CIDNP from the $\beta-\mathrm{CH}_{2}$
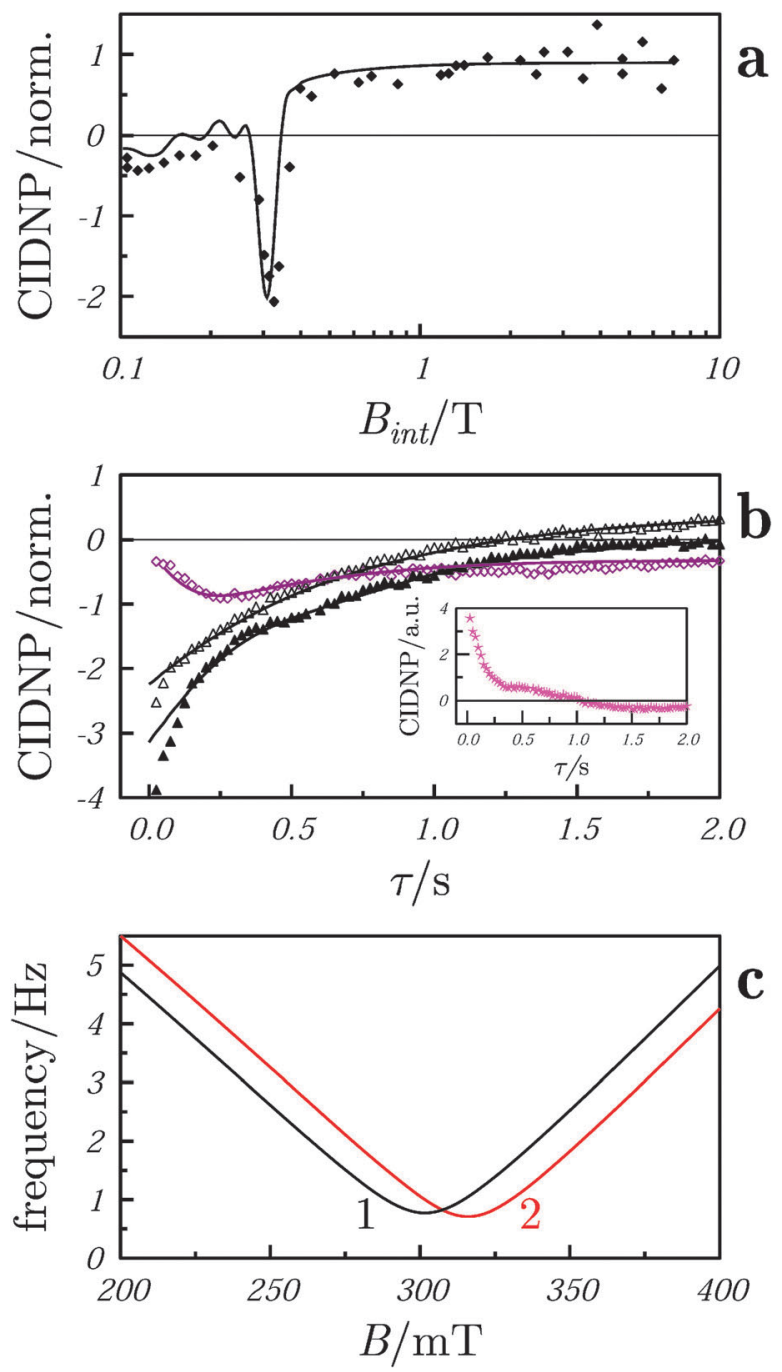

Fig. 4 CIDNP transfer in Subsystem 1 of $N$-Ac-Trp. In subplot (a) CIDNP of the $\alpha-\mathrm{CH}$ proton is shown as function of the $B_{\text {int }}$ field. Subplot (b) shows time dependence of CIDNP transfer between $\alpha-\mathrm{CH}$ (purple diamonds) $\beta 1-\mathrm{CH}_{2}$ (filled triangles) and $\beta 2-\mathrm{CH}_{2}$ (open triangles) at $B_{\text {int }}=313 \mathrm{mT}$; the inset shows the difference between CIDNP of the $\alpha-\mathrm{CH}$ and $\beta 1-\mathrm{CH}_{2}$ protons. Subplot (c) shows calculated energy differences (frequencies) for pairs of crossing levels $\beta \alpha \alpha \alpha-\alpha \alpha \beta \alpha$ (curve 1) and $\beta \alpha \alpha \beta-\alpha \alpha \beta \beta$ (curve 2) as a function of magnetic field. Theoretical calculations in (a) and (b) are shown by solid lines; protons are listed in the order $\left(\beta 1-\mathrm{CH}_{2}, \beta 2-\mathrm{CH}_{2}, \alpha-\mathrm{CH}, \mathrm{H} 2\right)$. protons. CIDNP as a function of time in this subsystem at a magnetic field of $313 \mathrm{mT}$, which corresponds to the position of the dip, is shown in Fig. $4 \mathrm{~b}$. Despite fast relaxation of this subsystem and low oscillation frequency it can be seen that there is a selective coherent exchange of CIDNP between the $\beta 1-\mathrm{CH}_{2}$ and $\alpha-\mathrm{CH}$ protons, while the polarization of the $\beta 2-\mathrm{CH}_{2}$ decays exponentially. Oscillations can be most clearly seen from the inset in Fig. $4 \mathrm{~b}$.

CIDNP transfer at a specific magnetic field as well as the transfer selectivity are both accounted for as a LAC effect. At a field of $313 \mathrm{mT}$ there is a LAC in the three-spin system consisting of the $\beta 1-\mathrm{CH}_{2}, \beta 2-\mathrm{CH}_{2}$, and $\alpha-\mathrm{CH}$. Addition of the $\mathrm{H} 2$ proton with slightly different couplings to the $\beta-\mathrm{CH}_{2}$ protons results in a small splitting of the LAC into two LACs with slightly different positions (Fig. 4c). In each case the LAC corresponds to the minimum in the curves in Fig. 4c.

At first glance, the selectivity of CIDNP transfer, which predominantly involves only one of the $\beta-\mathrm{CH}_{2}$ protons, is surprising because at low $B_{\text {int }}$ fields the $\beta-\mathrm{CH}_{2}$ protons are coupled strongly with their chemical shifts becoming practically non-distinguishable. However, selectivity is possible because in our experiments using a moderate rate of field variation only a single ZQC is excited (Fig. 5). As a consequence, field variation is non-adiabatic only for one pair of levels, while for the other levels it is adiabatic. The ZQC, which is responsible for the CIDNP transfer, is the coherence between the crossing levels, which correspond to the $\beta \alpha \alpha$ - and $\alpha \alpha \beta$-states at high field where the protons are weakly coupled (here the states are specified in the order $\left.\beta 1-\mathrm{CH}_{2}, \beta 2-\mathrm{CH}_{2}, \alpha-\mathrm{CH}\right)$. For these two levels the spin state of the $\beta 1-\mathrm{CH}_{2}$ proton changes (together with that of the $\alpha-\mathrm{CH}$ proton), while the state of the $\beta 2-\mathrm{CH}_{2}$ proton is the same for both levels. This fact gives transfer selectivity: due to mixing at the LAC the spins of the $\beta 1-\mathrm{CH}_{2}$ and $\alpha-\mathrm{CH}$ protons flip-flop, while the spin state of the $\beta 2-\mathrm{CH}_{2}$ proton stays unchanged. Due to the preparation (negative net

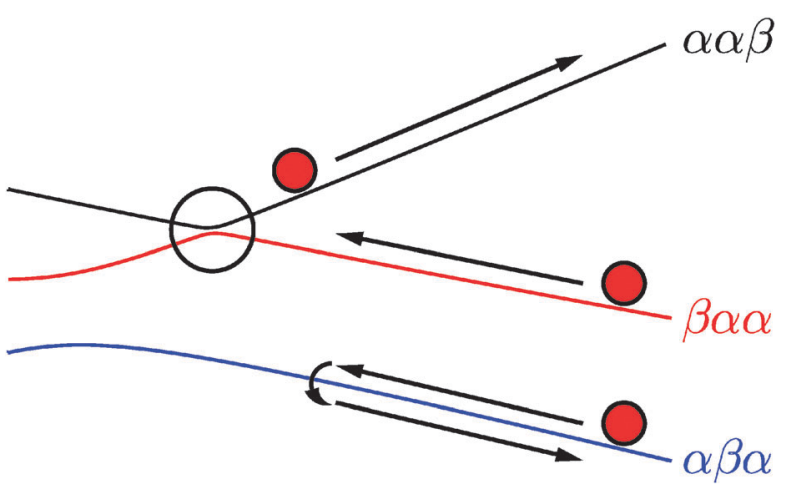

Fig. 5 Scheme explaining the selectivity of polarization transfer in the threespin system composed of $\beta 1-\mathrm{CH}_{2}, \beta 2-\mathrm{CH}_{2}$ and $\alpha-\mathrm{CH}$ protons. Initially the $\alpha \beta \alpha$ and $\beta \alpha \alpha$ states are overpopulated (state populations are shown by balls). After going to the LAC point and back the population of the $\alpha \beta \alpha$ state remains unchanged because for this state the field variation is adiabatic due to the fact that no LACS involving this state are passed. For the other two states the populations can be exchanged by coherent mixing at the LAC point enabling transfer of negative CIDNP from the $\beta 1-\mathrm{CH}_{2}$ proton to the $\alpha-\mathrm{CH}$ proton. 
CIDNP of the $\beta-\mathrm{CH}_{2}$ protons) the $\beta \alpha \alpha$-state is initially overpopulated. Since other ZQCs are not excited there is a single polarization pathway, which allows one to shift the negative CIDNP from the $\beta 1-\mathrm{CH}_{2}$ proton selectively to the $\alpha-\mathrm{CH}$ proton. In fact, polarization is transferred only within a pair of protons, which have the smallest difference in chemical shifts $\left(\beta 1-\mathrm{CH}_{2}\right.$ and $\alpha-\mathrm{CH}$ in our case). This is because the high-field states are grouped in the order of chemical shifts. For instance, for the manifold of states with $I_{z}=1 / 2$ the energies of states increase with increasing chemical shift of the protons in the spin-down state. This gives the order of high-field states shown in Fig. 5 and results in the selective CIDNP transfer between the $\beta 1-\mathrm{CH}_{2}$ and $\alpha-\mathrm{CH}$ protons.

Now let us describe the results obtained for the protons in the six-membered ring of $N$-Ac-Trp, which constitute Subsystem 2. The experimental field dependence of CIDNP transfer efficiency between the aromatic protons is shown in Fig. 6a. It is readily seen that at magnetic fields below $200 \mathrm{mT}$ the $\mathrm{H} 4$ and $\mathrm{H} 7$ protons exchange polarizations; at the same time, the H5 and H6 show almost no effects of polarization transfer. This observation is rather perplexing, since the direct $J$-coupling between the $\mathrm{H} 4$ and $\mathrm{H} 7$ protons (approximately $1 \mathrm{~Hz}$ ) is much weaker than the vicinal couplings of the aromatic protons including $\mathrm{H} 5-\mathrm{H} 6$ (approximately $7 \mathrm{~Hz}$ ). Nonetheless, the transfer is most efficient between the remote $\mathrm{H} 4$ and $\mathrm{H} 7$ protons.
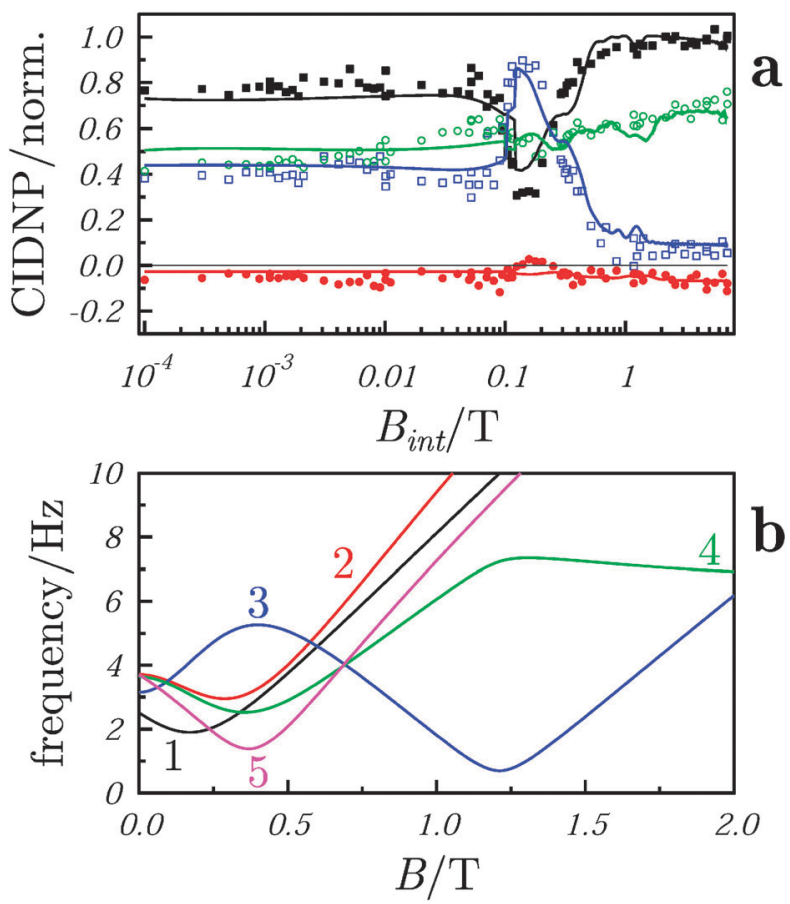

Fig. 6 Dependence of CIDNP on $B_{\text {int }}$ (a) and energy differences (in frequency units) (b) for different pairs of spin eigen-states shown for Subsystem 2 of $\mathrm{N}$-AcTrp. In subplot (a) we show CIDNP of protons H4 (filled squares, black), H5 (filled circles, red), H6 (open circles, green), H7 (open squares, blue); solid lines show results of theoretical calculation. In subplot (b) frequencies are shown for pairs of states $\alpha \alpha \alpha \beta-\beta \alpha \alpha \alpha$ (curve 1), $\alpha \beta \alpha \beta-\alpha \alpha \beta \beta$ (curve 2), $\alpha \alpha \beta \beta-\beta \beta \alpha \alpha$ (curve 3), $\beta \beta \alpha \alpha-$ $\beta \alpha \beta \alpha$ (curve 4), $\alpha \beta \beta \beta-\beta \beta \beta \alpha$ (curve 5); spins are listed in the order $(\mathrm{H} 4, \mathrm{H} 5, \mathrm{H} 6, \mathrm{H} 7$ ).
To understand this phenomenon it is of importance to have a closer look at the energy levels in this system and their avoided crossings. The energy levels of interest are shown in Fig. 6b. There are several LACs in this system that are expected to affect spins in different ways. Indeed, when the adiabatic levels are assigned with high-field spin states it turns out that for each pair of levels either the $\mathrm{H} 4-\mathrm{H} 7$ or the $\mathrm{H} 5-\mathrm{H} 6$ protons simultaneously exchange their projections giving selectivity of the transfer. For instance, for the pair of $\alpha \alpha \alpha \beta-\beta \alpha \alpha \alpha$ states the spins of the $\mathrm{H} 4$ and $\mathrm{H} 7$ protons exchange their projections, while for the pair of states $\alpha \beta \alpha \beta-\alpha \alpha \beta \beta$ the $\mathrm{H} 5$ and $\mathrm{H} 6$ protons do so. Here the states of this four-spin system are assigned in the following order: ( $\mathrm{H} 4, \mathrm{H} 5, \mathrm{H} 6, \mathrm{H} 7)$. The only exception is given by the pair of levels $\alpha \alpha \beta \beta-\beta \beta \alpha \alpha$ (see curve 3 in Fig. 6) where all states change giving CIDNP transfer in both pairs of spins, i.e., $\mathrm{H} 4-\mathrm{H} 7$ and $\mathrm{H} 5-\mathrm{H} 6$. Our theoretical calculation has revealed that ZQCs are predominantly excited for LACs, which involve simultaneous flips of the $\mathrm{H} 4$ and $\mathrm{H} 7$ protons. Simultaneous flips of the $\mathrm{H} 5$ and $\mathrm{H} 6$ protons occur with lower efficiency whereas other flip-flop transitions are practically absent.

In Fig. 7 CIDNP spectra of the aromatic region of $N$-Ac-Trp are shown for different delays $\tau$ together with the time evolution of polarization. From the spectra it is clearly seen that $\mathrm{H} 4$ and $\mathrm{H} 7$ efficiently exchange polarization, whereas all other NMR signals vary only slightly during the transfer process. The polarization transfer is coherent, as is clear from the oscillations in the time dependence; the theoretical calculation
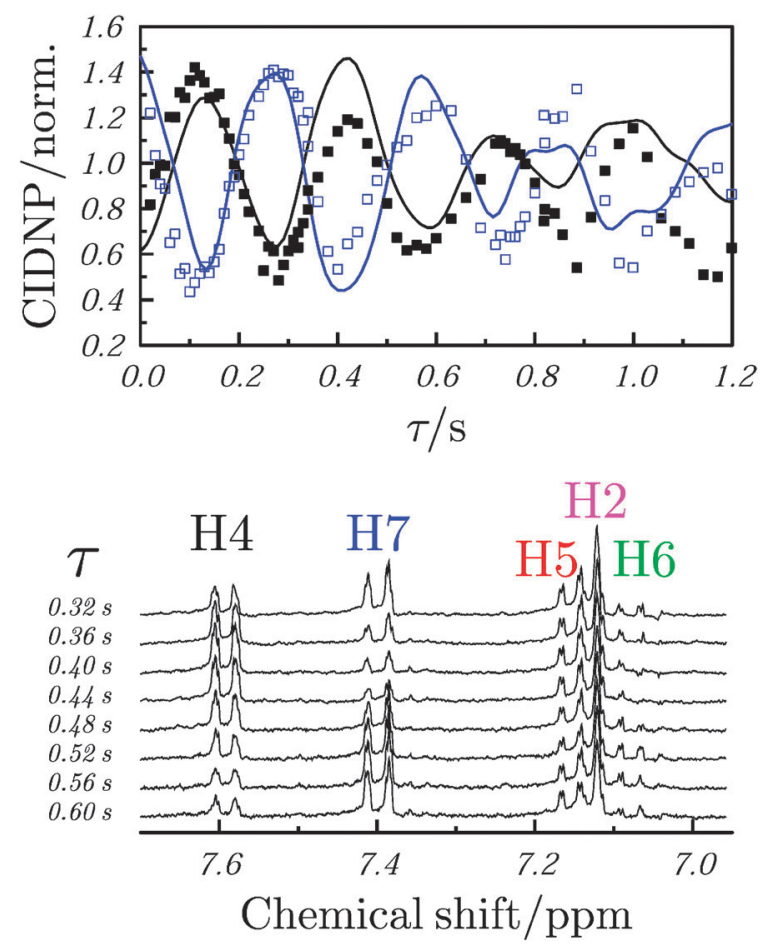

Fig. 7 Time-dependence of CIDNP transfer (top) between the $\mathrm{H} 4$ (filled squares, black) and $\mathrm{H} 7$ (open squares, blue) protons at $B_{\text {int }}=157 \mathrm{mT}$; the aromatic part of the experimental CIDNP spectra is also shown (bottom) for different values of the delay $\tau$. Solid lines show results of theoretical calculation. 
is in good agreement with the data. We also studied (see Fig. S2, ESI $\dagger$ ) the time-dependence of CIDNP transfer at different strengths of the $B_{\text {int }}$ field. In each case we obtained predominantly polarization transfer between the $\mathrm{H} 4$ and $\mathrm{H} 7$ protons; polarization transfer between the $\mathrm{H} 5$ and $\mathrm{H} 6$ protons was also found but its efficiency was lower. This can be seen from the amplitude of oscillations in Fig. S2 (ESI $\dagger$ ): at all fields this amplitude is considerably higher (a factor of 3-5) for the $\mathrm{H} 4-\mathrm{H} 7$ protons than for the $\mathrm{H} 5-\mathrm{H} 6$ protons. CIDNP transfer in other pairs of spins was not found. Thus, LACs impose the transfer selectivity in Subsystem 2 as well. At low fields the entire system exhibits collective coherent evolution involving all spins, no matter whether they are coupled directly or only indirectly. As in the case of Subsystem 1 CIDNP transfer mediated by LACs is efficient between the spins with the closest chemical shifts, i.e., in pairs $\mathrm{H} 4-\mathrm{H} 7$ and $\mathrm{H} 5-\mathrm{H} 6$. In addition to CIDNP transfer we studied the related phenomenon of longitudinal relaxation of the protons of $\mathrm{N}$-Ac-Trp. Strong coupling of spins has a pronounced effect on the observed field dependence of the $T_{1}$-relaxation times (relaxation dispersion curves). It is worth noting that for a small molecule like $N$-Ac-Trp we are always in the fast motional regime; thus, in the dispersion curves no features arise from the variation of the motional regime at $\omega=$ $\gamma \cdot B_{\text {int }}=1 / \tau_{\mathrm{c}}$ because the motional correlation time, $\tau_{\mathrm{c}}$, is too short. In $N$-Ac-Trp we see not only the two coupled four-spin systems discussed above, but, in addition, there are the $\mathrm{CH}_{3}$ protons. Their $T_{1}$-relaxation time is field-independent (Fig. S3, ESI $\dagger$ ) because they are completely decoupled from the rest of the molecule. In contrast to this trivial observation, in the coupled proton subsystems we see pronounced features in the relaxation dispersion. In both networks spins relax with a common $T_{1}$ at low fields. In Subsystem 1 there are sharp features caused by LACs (Fig. S3, ESI $\dagger$ ). Thus, strong coupling and LACs significantly affect both coherent and incoherent spin dynamics.

\section{Conclusion}

In this work we have analyzed polarization transfer among strongly coupled spins in NMR experiments with fast fieldcycling. In particular, we have shown that such a transfer has a coherent nature, thus being fast, efficient, selective and controllable. We have found that polarization transfer occurs via ZQCs that are excited without NMR pulses by means of a sudden field variation. Coherent effects clearly manifest themselves in the time dependence of polarization, which contains a pronounced oscillatory contribution. We have described in detail the coherent pathways of polarization transfer and elucidated the role of LACs in the observed phenomena. Good agreement between experimental results and theoretical calculations allowed finding particular coherences responsible for the polarization transfer, and accounting for the transfer selectivity. We have also demonstrated that exploiting LACs opens a way for selective population exchange between particular pairs of spin energy levels and thus for polarization transfer between well-defined spin orders. By mapping out
LACs one can also explain coherent spin evolution of complex systems and predict transfer pathways. Thus, the LAC concept is extremely helpful for interpreting experimental data, manipulating spin hyperpolarization and designing new experiments. Typically, evolution of strongly coupled spin systems can be addressed only numerically; however, LACs allow identifying the dominating transfer pathways in a simple and straightforward way. As a further advantage of the LAC methodology one should note that computational efforts needed to identify LACs in the system (i.e., solving the eigen-problem for the Hamiltonian) are, in fact, much less than those required to calculate the full spin evolution.

It is worth noting that LACs can also be used for polarization transfer among hetero-nuclei. The most obvious case of such transfer is given by the situation where protons and heteronuclei are coupled strongly and exchange their net magnetizations; however, this requires going to ultralow fields $(10 \mu \mathrm{T}$ or even lower), which is technically rather demanding. An alternative is given by LACs occurring at magnetic fields ${ }^{27,28}$ where (i) protons are coupled strongly and (ii) have unequal couplings to the hetero-nucleus. The LAC positions then depend on the spin state of the hetero-nucleus, therefore by performing proton-proton polarization transfer at such LACs it is possible to hyperpolarize also the hetero-nucleus. Although the polarization of the hetero-nucleus formed will have a multiplet but not net character, its NMR lines will be strongly enhanced.

Although in our experiments non-thermal polarization has been prepared by CIDNP our conclusions are valid for other kinds of hyperpolarization as well, most notably, for PHIP, in which case polarization transfer phenomena are common ${ }^{13,40-43}$ at low field. It is worth noting that although we studied only scalar coupled spins $1 / 2$ our results are more general. In fact, other interactions (for instance, residual dipolar couplings in protein and liquid crystalline samples) can lead to similar polarization transfer effects. The mechanisms considered are always operative when (i) a polarized spin system goes to the strong coupling regime upon field variation and (ii) the field switch is non-adiabatic. In addition, pronounced effects conditioned by LACs are expected for any kind of spin-spin interaction. A related phenomenon is the indirect detection of the quadrupolar ${ }^{14} \mathrm{~N}$ nuclei ${ }^{44,45}$ by observing the proton-nitrogen polarization transfer at LAC points. Although in these experiments usually a relaxation-type mechanism for polarization exchange is postulated, ${ }^{44,45}$ our results show that it is also possible that polarization is transferred via spin coherences. ${ }^{46}$ Our study opens additional opportunities for spectroscopic and dynamic studies of coupled spin systems. First, the study of polarization transfer and assignments of LACs gives additional spectroscopic information, since the transfer frequencies and LAC positions are uniquely related to the NMR parameters of a spin system. Second, ZQCs can be long-lived, ${ }^{4-50}$ allowing determination of very small $J$-couplings.

In summary, our study shows the importance of coherent phenomena in NMR experiments utilizing fast field-cycling techniques. The analysis based on LACs is general and can be applied to other experiments of non-thermally polarized spin 
systems as well. In addition, study of coherent polarization transfer effects potentially provides new spectroscopic and dynamic information on the system under study.

\section{Acknowledgements}

This work has been supported by the Russian Foundation for Basic Research (projects 11-03-00296a, 12-03-31042, 12-0333082, 13-03-00437), Research Group Linkage Program of the Alexander von Humboldt Foundation, EU-COST Action TD1103, the Program of the Division of Chemistry and Material Science RAS (project 5.1.1) and the program of Russian Government P220 (agreement no.11.G34.31.0045).

\section{Notes and references}

1 T. Prisner and W. Köckenberger, Appl. Magn. Reson., 2008, 34, 213-218.

2 M. Borghini, W. De Boer and K. Morimoto, Phys. Lett. A, 1974, 48, 244-246.

3 K. H. Hausser and D. Stehlik, Adv. Magn. Reson., 1968, 79-139.

4 C. D. Jeffries, Dynamic Nuclear Orientation, Interscience, New York, 1963.

5 K. M. Salikhov, Y. N. Molin, R. Z. Sagdeev and A. L. Buchachenko, Spin Polarization and Magnetic Effects in Chemical Reactions, Elsevier, Amsterdam, 1984.

6 K. Münnemann and H.-W. Spiess, Nat. Phys., 2011, 7, 522-523.

7 J. Natterer and J. Bargon, Prog. Nucl. Magn. Reson. Spectrosc., 1997, 31, 293-315.

8 R. A. Green, R. W. Adams, S. B. Duckett, R. E. Mewis, D. C. Williamson and G. G. R. Green, Prog. Nucl. Magn. Reson. Spectrosc., 2012, 67, 1-48.

9 M. S. Albert, G. D. Cates, B. Driehuys, W. Happer, B. Saam, C. S. Springer and A. Wishnia, Nature, 1994, 370, 199.

10 J. H. Ardenkjaer-Larsen, B. Fridlund, A. Gram, G. Hansson, L. Hansson, M. H. Lerche, R. Servin, M. Thaning and K. Golman, Proc. Natl. Acad. Sci. U. S. A., 2003, 100, 10158.

11 S. E. Day, M. I. Kettunen, F. A. Gallagher, D. E. Hu, M. Lerche, J. Wolber, K. Golman, J. H. Ardenkjaer-Larsen and K. M. Brindle, Nat. Med., 2007, 13, 1382.

12 T. Maly, G. T. Debelouchina, V. S. Bajaj, K.-N. Hu, C.-G. Joo, M. L. Mak-Jurkauskas, J. R. Sirigiri, P. C. A. van der Wel, J. Herzfeld, R. J. Temkin and R. G. Griffin, J. Chem. Phys., 2008, 128, 052211.

13 T. Theis, P. Ganssle, G. Kervern, S. Knappe, J. Kitching, M. P. Ledbetter, D. Budker and A. Pines, Nat. Phys., 2011, 7, 571-575.

14 K. H. Mok, L. T. Kuhn, M. Goez, I. J. Day, J. C. Lin, H. N. Andersen and P. J. Hore, Nature, 2007, 447, 106-109.

15 M. Renault, S. Pawsey, M. P. Bos, E. J. Koers, D. Nand, R. Tommassen-van Boxtel, M. Rosay, J. Tommassen, W. E. Maas and M. Baldus, Angew. Chem., Int. Ed., 2012, 51, 2998-3001.
16 J. A. Aguilar, P. I. P. Elliott, J. Lopez-Serrano, R. W. Adams and S. B. Duckett, Chem. Commun., 2007, 1183-1185.

17 L. Frydman and D. Blazina, Nature, 2007, 3, 415.

18 J. von Neumann and E. Wigner, Phys. Z., 1929, 30, 467.

19 J. Colpa and D. Stehlik, Chem. Phys., 1977, 21, 273-288.

20 D. Stehlik and J. Colpa, Chem. Phys., 1977, 21, 289-299.

21 T. R. Eichhorn, M. Haag, B. van den Brandt, P. Hautle and W. T. Wenckebach, Chem. Phys. Lett., 2012, 555, 296.

22 F. Mentink-Vigier, Ü. Akbey, Y. Hovav, S. Vega, H. Oschkinat and A. Feintuch, J. Magn. Reson., 2012, 224, 13.

23 K. R. Thurber and R. Tycko, J. Chem. Phys., 2012, 137, 084508.

24 K. Miesel, K. L. Ivanov, A. V. Yurkovskaya and H.-M. Vieth, Chem. Phys. Lett., 2006, 425, 71-76.

25 L. Buljubasich, M. B. Franzoni, H. W. Spiess and K. Münnemann, J. Magn. Reson., 2012, 219, 33.

26 M. B. Franzoni, L. Biljubasich, H.-W. Spiess and K. Münnemann, J. Am. Chem. Soc., 2012, 134, 10393.

27 A. S. Kiryutin, K. L. Ivanov, A. V. Yurkovskaya, R. Kaptein and H.-M. Vieth, Z. Phys. Chem., 2012, 226, 1343.

28 S. E. Korchak, K. L. Ivanov, A. N. Pravdivtsev, A. V. Yurkovskaya and H.-M. Vieth, J. Chem. Phys., 2012, 137, 094503.

29 A. S. Kiryutin, S. E. Korchak, K. L. Ivanov, A. V. Yurkovskaya and H.-M. Vieth, J. Phys. Chem. Lett., 2012, 3, 1814.

30 O. B. Morozova, K. L. Ivanov, A. S. Kiryutin, R. Z. Sagdeev, T. Köchling, H.-M. Vieth and A. V. Yurkovskaya, Phys. Chem. Chem. Phys., 2011, 13, 6619.

31 S. Grosse, A. V. Yurkovskaya, J. Lopez and H.-M. Vieth, J. Phys. Chem. A, 2001, 105, 6311.

32 F. J. J. De Kanter and R. Kaptein, Chem. Phys. Lett., 1979, $62,421$.

33 K. L. Ivanov, A. V. Yurkovskaya and H.-M. Vieth, J. Chem. Phys., 2008, 128, 154701.

34 A. Messiah, Quantum mechanics, North-Holland Publishing Company, Amsterdam, 1965.

35 A. I. Chichinin, J. Phys. Chem. B, 2013, 117, 6018.

36 S. E. Korchak, A. S. Kiryutin, K. L. Ivanov, A. V. Yurkovskaya, Y. A. Grishin, H. Zimmermann and H.-M. Vieth, Appl. Magn. Reson., 2010, 37, 515-537; and ESI therein.

37 O. W. Sørensen, G. W. Eich, M. H. Levitt, G. Bodenhausen and R. R. Ernst, Prog. Nucl. Magn. Reson. Spectrosc., 1984, 16, 163-192.

38 M. Carravetta and M. H. Levitt, J. Am. Chem. Soc., 2004, 126, 6228.

39 G. Pileio, Prog. Nucl. Magn. Reson. Spectrosc., 2010, 56, 217. 40 K. D. Atkinson, M. J. Cowley, P. I. P. Elliott, S. B. Duckett, G. G. R. Green, J. Lopez-Serrano and A. C. Whitwood, J. Am. Chem. Soc., 2009, 131, 13362.

41 S. Gloggler, R. Müller, J. Colell, M. Emondts, M. Dabrowski, B. Blümich and S. Appelt, Phys. Chem. Chem. Phys., 2011, 13, 13759-13764.

42 T. Theis, M. P. Ledbetter, G. Kervern, J. W. Blanchard, P. J. Ganssle, M. C. Butler, H. D. Shin, D. Budker and A. Pines, J. Am. Chem. Soc., 2012, 134, 3987-3990.

43 S. E. Korchak, K. L. Ivanov, A. V. Yurkovskaya and H. M. Vieth, Phys. Chem. Chem. Phys., 2009, 11, 11146. 
44 R. Kimmich, Z. Naturforsch., A: Phys. Phys. Chem. Kosmophys., 1977, 32, 544.

45 F. Winter and R. Kimmich, Mol. Phys., 1982, 45, 33.

46 E. P. Sunde and B. Halle, J. Magn. Reson., 2010, 203, 257.

47 G. Pileio, M. Carravetta and M. H. Levitt, Phys. Rev. Lett., 2009, 103, 083002.
48 R. Sarkar, P. Ahuja, P. R. Vasos and G. Bodenhausen, Phys. Rev. Lett., 2010, 104, 053001.

49 R. Sarkar, P. Ahuja, P. R. Vasos, A. Bornet, O. Wagnieres and G. Bodenhausen, Prog. Nucl. Magn. Reson. Spectrosc., 2011, 59, 83-90.

50 A. Bornet, S. Jannin, J. A. Konter, P. Hautle, B. van den Brandt and G. Bodenhausen, J. Am. Chem. Soc., 2011, 133, 15644. 